\title{
Implementing Web 2.0 Technologies in Mining Engineers Language Communications
}

\author{
Alena Perevalova ${ }^{1, *}$ \\ ${ }^{1}$ Kemerovo State University, Institute of Philology, Foreign Languages and Media Communication, \\ Department of Foreign Languages, 650000 Kemerovo, 6 Krasnaya st., Russian Federation
}

\begin{abstract}
This paper is devoted to the author's experience of applying the most popular Web 2.0 technologies to mining ingeneers' educational process. The paper deals with incorporating such services as RSS, weblogs, microblogs, podcasts, mashups, wikis into the process of teaching English to future mining specialists. With the development of globalization processes, higher education in mineral resource universities has new objectives, i.e. training professionals capable of working effectively in the changed conditions of the global mining industry. Modern higher educational system has a number of specific features and requires certain changes in the content and organizing the teaching process. Web 2.0 opens up great opportunities for educational practice: the use of free electronic resources used for training purposes; creation of network content; interpersonal interaction of all the members of the educational process, etc. The paper notes the effectiveness of implementing some Web 2.0 technologies to make education of mining engineers more realistic, increase their motivation to become ready for professional communications with foreign colleagues, which will help to raise mining safety and productivity.
\end{abstract}

\section{Introduction}

The demand of competitive, socially mobile, highly professional specialists, aimed at personal growth and self-development, encourages higher educational establishments to develop and integrate innovative teaching technologies as well as to adapt modern online technologies for training higher education graduates. In contrast with the traditional World Wide Web, which presented the medium for getting information, Web 2.0 offers the platform for social interaction [1-4]. Owing to implementing software innovations it has become possible to shift the emphasis from computer and media technologies to communication, content-sharing and collaboration. Using Web 2.0 technologies in teaching a foreign language to future mining engineers promotes favourable conditions for increasing students' motivation, doing scientific research, realizing cooperative projects, developing communication and social skills.

\footnotetext{
* Corresponding author: aljona 04@mail.ru
} 


\section{Materials and Methods}

The phenomena of social networks or Web 2.0 emerged in 2000-2001, and the term itself widely spread due to the published Tim O'Reilly's article "What Is Web 2.0" [5]. Unlike Web 1.0 , which emphasized mostly technologies, the key factor in Web 2.0 is a person, his social communication and personal preferences.

Adapted for educational process Web 2.0 services allow each student to:

- post their own thoughts, make notes, comment blog posts (such sites, as www.researchgate.net, http://www.linkedln.com were used);

- create and keep account on mining engineers' association site: http://agi-portal.ru/);

- design encyclopedias for mining engineers directly from a web browser (some examples of used websites are http://www.wikispaces.org, http://www.wikipedia.org);

- share pictures, photos, video files, describing different sides of underground and open-pit mining (http://www.facebook.com);

- record audio files, keep and distribute them online (the students used http://podomatic.com); post text files, slides, charts and drafts of mining works (with the help of such websites, as http://www.orkut.com);

- create a virtual personality to promote personal and professional skills (it was possible on such websites as: http://www.facebook.com, http://www.linkedin.com);

- exchange messages, ideas, articles, news (using RSS feeds);

- create and modify text report files (http://docs.google.com) and diagrams (http://www.gliffy.com).

All used and embedded into the teaching process Web 2.0 services have five main technological characteristics. Firstly, these are technologies based on AJAX (Asynchronous JavaScript and XML), which present a new approach to building interactive user interfaces for web applications. It allows to update the page content dynamically and synchronously: the page itself loads needed data without complete reloading.

Secondly, using RSS (Really Simple Syndication) lets the students place information on a large number of websites simultaneously. The RSS newsreader examines each feed several times per hour in order to find out if new content is added or replaced, and to report this modification to the user $[2,6,8,12]$. The great benefit of using RSS in the educational process is the possibility for students not to visit a web page in order to get new content. So, it considerably accelerates the process of information exchange. RSS is actively used in teaching students both for their individual studies and for cooperative research projects on shared blogs.

As the third technological characteristic of Web 2.0, open social web services API (Application Programming Interfaces) should be mentioned. They represent a set of methods that can be used to access features or internal functions of other programs. The openness of API allows any user to create 'hybrid services' (mash-up). Mashup represents a website or a web application combining the information from different sources $[2,7,12]$. The leaders of popular hybrid services are Google and a bit less known Flickr.com, which link together various content from a wide range of different sources by using fragments of pre-built programming modules. Moreover, mashup approach provides favourable conditions and influences for e-learning by creating web-based tools and learning content.

Furthermore, one more technological characteristic of all Web 2.0 services is availability of constant links to earlier published data that lets follow individual and group backstories. At last, using tags, i.e. key words that characterize particular load of information, is a distinguishing feature of many Web 2.0 services. Unlike Web 1.0, where the organization of information was carried out by placing it in catalogs, Web 2.0 uses tags in order to organize data. Due to this peculiarity of information delivery and arrangement, a new term appeared folksonomy, i.e. a new form of metadata; collaborative categorizing information (links, photo, 
video clips, etc.) by means of randomly selected notes (tags) [10].

Additionally, some social features of Web 2.0, which we took into account in the teaching process, should be mentioned. They can be formulated as follows:

- openness;

- availability of mass, anonymous, segmented audience promoting self-actualization and selfrealization;

- users' participation in developing internet resources or services and control over them; decentralization and communication of large social groups;

- increasing users' engagement: from collective intelligence to cooperative intelligence.

In the age of information overload it is very important for a student to create some social network, which gives access to necessary resources including both data and contacts when it is needed. It means that rapid speed of social services development has required restructuring all the units of educational system that is perspectives, objectives syllabus, teaching techniques, and learning activity forms.

Thus, Web 2.0 services have broken new ground for network members' interaction, i.e. nowadays there is a unique opportunity for a group communication on the one hand, and a great chance for the creation of virtual personality and its adaptive management on the other hand. The key element of any social network is its users and their interests. As we consider educational process, students' interests and needs are taking center stage.

Secondly, new web services have evolved from information storage into a convenient platform, i.e. an approach to solving a range of tasks. Web 2.0 services have opened up new possibilities for the participants of the educational process, as they let collectively create educational content by means of group interaction and on condition that there is a radical decentralization along with complete confidence in all the participants of educational process.

Thirdly, owing to ease of use and low price currently existing technologies of the Internet allow not only create, store and arrange information in any format (video and audio files, animation, text files, etc.), but also exchange and display it, get comments and critical feedback, that is also extremely significant for the educational process.

Furthermore, unique RSS technologies help students cope with wealth of information and, therefore, with the necessity of improving their ability to read quickly. Our students learnt to analyse the information in order to understand which was worth reading, and to switch to careful analysis of obtained information. RSS has let the students control the content of the Internet reducing the time for browsing the sites, they are interested in, in order to check updates. They have got an opportunity to receive the information about content updates that allows to filter out irrelevant information thanks to displaying the headings of publications.

Audio podcasting was used for recording some lectures and delivering them to students, who, in their turn, could reproduce these audio files directly on the website or download them on a computer or mp3 player. Courses or lectures in the form of podcasts let the students play them not only at home, but any place in any time. Additionally, audio podcasts were used to practice students' listening skills in English, as they were also recorded by native speakers $[2,11,13]$.

As for the types of podcasts used in the teaching process by us there should be mentioned the following: 1) podcasts for learning new vocabulary (a lecturer explains the word meaning, gives word combinations or idioms, and illustrates context with these words/phrases); 2) podcasts aimed at developing listening skills and including listening tasks; 3) podcasts designed for the whole English lesson (such lessons and podcasts should be provided with hand-outs). Audio podcasting services served not only as a means of developing listening skills, but also as a tool of improving speaking skills.

Podcasting has a range of advantages over traditional educational forms. This 
technology lets listen to and look through learning materials in any place and any time, allocating study time in the most convenient way. Podcasts can be listened to as many times as it is needed, in whole or in parts. In general, students can set the most appropriate learning pace for them.

Microblogs being one of the forms of social networks have gained great popularity among educators due to the fact that they are more interactive than blogs, involve more number of users, are of a more informal nature, and can serve as a tool of professional communication. They let ask questions and get responses from lots of users immediately, exchange links to Internet resources, information about conferences/ seminars etc. Messages in microblogs (e.g. Twitter, Plurk) are limited in number of printed characters and can be delivered to other users in a variety of ways, including text messages, instant messages, e-mail, MP3, etc.

A weblog is a social network that perfectly suits the didactic purposes as it allows to keep and categorize necessary and excess information in any forms (diagrams, charts, pictures, photos, video and audio files); to create open and closed communities for discussing problems and tasks, for realizing group projects, for controlling how well students took in learning information with the help of online tests, surveys and discussions, reviews, etc. $[2,8,9,12]$.

Weblogs can be categorized into teacher's blogs, individual blogs, and collective blogs. In a teacher's blog the subject information is placed. It can include the curriculum, the material studied in a particular lesson, class assignment, a list of recommended resources for further and follow-up study, links to online tests for self-study. While working with a teacher's blog students develop the ability to hold discussions on professional topics, reading skills in a foreign language.

An individual blog can act as a student electronic portfolio containing a collection of materials which demonstrate personal progress over a definite period of time. When working with an individual educational blog, students develop writing skills; reading skills: the ability to extract necessary information; evaluate the importance of information [13, 14]. Besides, students learn to use the necessary language tools for self-presentation, to represent their country and culture in a foreign language environment, to express their opinion, agreement/ disagreement when commenting. Individual blogs can also include personal details: information about family, friends, interests, achievements, photos and video clips.

A collective blog is designed for organizing extracurricular work in a group. On such blog a teacher posts assignments, helpful information, materials for commenting (text, audio files and video clips). Unlike individual blogs, on collective educational weblogs students' comments are placed sequentially on the same page. This greatly facilitates learning about other students' opinions and stimulates discussions. Each participant is able to express his/her own point of view on a discussed issue, notice a different view, perceive cultural diversity as a norm of coexistence in the modern multicultural world, and develop the ability of critical thinking.

When organizing work on a collective blog it is essential to engage the maximum number of participants in discussions. While working on a collective blog students develop writing skills, namely, the ability to present the content of read or heard text in a foreign language in the form of brief messages; the ability to use the necessary linguistic means to express the opinion, agreement or disagreement in a tolerant way; the ability to draw an analogy, comparison, matching using available linguistic means; the ability to give arguments [13]. Moreover, students improve reading skills, such as the ability to find the necessary facts and information; separate significant information from less important; determine cause-and-effect link between events. 


\section{Results and Discussion}

Integrating Web 2.0 technologies in teaching English to future mining engineers has proven to be effective and inspiring experience both for students and teachers, and also has encouraged student-centered teaching, students' creativity, motivation and responsibility for the results of their work.

Using blogs for learning such speaking topics as "Mining methods", "Mining and Environment", "Economics and Mining" students have developed skills in searching, analyzing, and critical evaluation of information, working with information flows on the Internet, working individually and in groups, and analytical skills. Weblogs have also helped students promote communicative competence through active participation of members of educational community in different discussions.

Weblogs have given the students an opportunity to express their own opinion, promoted developing critical thinking, active stand and self-reflection. The students study their own learning style and thinking style. Having potential readership and receiving responses from it, the student is gradually moving from writing short reviews to creating more serious essays. So, weblogs have been used in teaching academic writing. Placing draft versions of essays in the blog gives the teacher the opportunity to trace the process of forming the main idea of the essay and the student's position on the subject under consideration. In an online discussion with groupmates and a teacher, a student has the possibility to make adjustments to the vision of the issue and the way of presenting the material.

Blogs have been used in teaching not only academic writing, but also business correspondence (i.e. writing official documents, business letters, office memo, etc.). In this way students acquire professional skills needed in professional life.

Additionally, educational weblogs have been used as a medium for posting publications. Students quickly realize that their messages can be read not only by teachers and classmates, but can be evaluated and commented by anyone. Posting on blogs is a legitimate opportunity to communicate with a real audience, to find like-minded people and to become a part of a special interest community. Over time, the blog becomes a virtual journal of the student's published written work (portfolio). Turning to the archive of their work students can easily find their previously posted material and assess the progress made when studying by comparing the latest essays with 'older' ones.

Microblogs have been used in the learning process as a means of:

- organizing students learning activity (sending out learning tasks, reminders, links to Internet resources);

- managing the learning process (efficient and operational communication, reminders, announcements);

- monitoring the activities of specialists in the field of interest (in this case, mining engineering);

- operational and interactive communication when working on a collective project.

Social networking services implemented in our educational process have helped the students create some academia and share resources, data, thoughts and experiences with each other. The main benefit of it was the possibility of effective interaction. The technology of creating and developing communities let teachers interact with several students at the same time online by means of instant messaging system. Using such social services as calendars allowed the students to plan their classes and meetings.

An outstanding feature of new technologies is also a possibility of developing and changing educational content by students. Educators working in the field of distant learning pointed out that using such tools as wiki-technologies and blogs students moved from discussing some topics with each other to discussing a wider range of themes with specialists all over the world. Furthermore, exchange of information and knowledge 
between students, teachers and specialists in mining engineering let create top-quality learning environment.

Web 2.0 technologies have brought about the availability of training programs, cataloguing and rating assessment, and lots of other crucial things to improve the availability and high quality of education. The educational course for mining engineers have been created as a compilation of educational material with a variety of audio files, video clips and links to different Internet resources for the students to be able to study it before the lesson. All these positive changes in the educational process have encouraged the improvement of the quality of received knowledge, students' awareness and expertise.

\section{Conclusion}

Due to the characteristics such as ease of use, availability, effectiveness of the information space organization, interactivity, multimedia possibilities, security and reliability, Web 2.0 technologies have considerable potential in forming intercultural competence of future specialists in mining engineering. This includes accelerating the speed of lessons and increasing motivation to learn foreign languages and cultures owing to visual presentation of educational material and enhancing its emotional component; ensuring efficiency of independent work by providing each student with the opportunity to choose the most appropriate way and pace of studying educational content; improving skills of productive cross-cultural interaction; providing operational access necessary information; knowledge of netiquette; skills to organize and hold discussions on professional topics by participating in online conversations, posting on weblogs, social networks, etc.

\section{References}

1. M.A. Chatti, D. Dahl, M. Jarke, WEBIST 2008, 4th International Conference on Web Information Systems and Technologies, 1, 370-375 (2008)

2. T. Orehovački, M. Konnecki, D. Radošević, 31st MIPRO International Convention on Computers in Education, 1, 269-273 ( 2008)

3. A. Darwish, K. I. Lakhtaria, Jour. Of Adv. In Inf. Tech., 2:4 (2011)

4. P. Gautam, Schol. Res. Jour. Hum. Scien. Eng. Lang, 1:3 (2014)

5. S. J. Cold, ACM SIGITE Newsletter, 3:1, 6-9 ( 2006)

6. B. Kieslinger, F. Wild, O. Arsun, 1 st European Conference on Technology Enhanced Learning, 1, 640-645 (2006)

7. D. Munca, Web 2.0 as Cognitive Tools in Teaching a Foreign Language (LAP, Los Angeles, 2010)

8. K. Placing, M-H Ward, M. Pead, P. T. Teixeira, UniServe Science Blended Learning Symposium Proceedings, 2, 159-164 (2005)

9. W. G Stock, Inf. Serv. Use, 27:3, 97-103 (2007)

10. J. P. Shim, J. Shropshire, S. Park, H. Harris, N. Campbell, Ind. Man. \& Data Syst., 107:4, $587-600$ (2007)

11. S. Tyagi, IJEDICT, 8:2, 28-43 (2012)

12. L. Lee, ReCALL, 22:2, 212-227 (2010)

13. L. Lee, Lang. Learn. \& Tech, 15:3, 87-109 (2011) 